\title{
SZEGÖ FUNCTIONS ON A LOCALLY COMPACT ABELIAN GROUP WITH ORDERED DUAL $\left({ }^{1}\right)$
}

\author{
BY \\ I. I. HIRSCHMAN, JR.
}

1. Introduction. Let $c(\theta) \in L^{1}(T)$ where $T$ is the group of real numbers modulo 1 , and let

$$
c(n)=\int_{T} c(\theta) e^{-2 \pi i n \theta} d \theta, \quad n \in I .
$$

Here $I$ is the group of integers. For several decades studies have been made of various properties of the finite section (Toeplitz) matrices

$$
[c(j-k)]_{j, k=0, \ldots, n}
$$

and the half-infinite (Wiener-Hopf) matrix

$$
[c(j-k)]_{j, k=0,1, \ldots} \text {. }
$$

See the book of Grenander and Szegö [6] for a survey of this subject up to 1958. In [9] and in the present paper, which is a continuation of [9], we have succeeded in placing part of this material in a rather general context. In this theory $T$ and $I$ are replaced by any pair of dual locally compact Abelian groups $\Theta$ and $\Xi$ for which a distinguished measurable order relation is given on $\Xi$. When $\Theta=T$ and $\Xi=I$ the theory reduces to a generalization of Baxter's Banach algebra presentation of Toeplitz forms and Szegö polynomials. See [1]-[3]. Since a rather extensive introduction to the present subject is given in [9] (for the case $\Theta$ compact) we will not pause here to outline the theory which follows.

2. Measurable orders. In this section we will define and study measurable order relations on a locally compact group $\Xi$. The simplest example is $\Xi=R_{1}$, the additive group of real numbers, with its usual order. A slightly more complicated example is $\Xi=R_{2}=R_{1} \times R_{1}$ with the lexicographic order, $\left(x_{1}, y_{1}\right)<\left(x_{2}, y_{2}\right)$ if $y_{1}<y_{2}$ or if $y_{1}=y_{2}$ and $x_{1}<x_{2}$.

We assume that a Haar measure $d \xi$ has been fixed on $\Xi . \mathscr{B}$ is the collection of all Borel sets on $\Xi$ (the smallest $\sigma$-ring containing all compact sets). A set $B$ is "Borelmeasurable" if $B \in \mathscr{B}$. A set $A$ is "measurable" if $A \cap B \in \mathscr{B}$ for every $B \in \mathscr{B}$.

Received by the editors January 8, 1965.

(1) This research was supported in part by the National Science Foundation under Grant No. GP-2089, and by the United States Air Force Office of Scientific Research under contract No. AF-AFOSR 721-65. 
A binary relation " $<$ " on $\Xi$ is a measurable order relation if it has the following properties:

(i) for each $\xi \in \Xi$ exactly one of $\xi=0, \xi>0, \xi<0$ holds;

(ii) $\xi_{1}<\xi_{2}$ and $\xi_{2}<\xi_{3} \Rightarrow \xi_{1}<\xi_{3}$;

(iii) $\xi_{1}<\xi_{2} \Rightarrow \xi_{1}+\eta<\xi_{2}+\eta$ for all $\eta \in \Xi$;

(iv) if $B \in \mathscr{B}$ then $\{\xi \in B \mid \xi>0\} \in \mathscr{B}$.

Property (iv) asserts that $\{\xi \mid \xi>0\}$ is measurable. It need not, however, be Borel measurable.

LEMMA 2a. If $\Xi$ is compact and has a measurable order relation $\Xi$ is trivial.

Proof. If $\Xi$ is not trivial there exists a Borel set $B$ of positive Haar measure such that $B<0$. Then $B+B<0$ and $B+B$ contains an open set $A, A<0$. Let $-a \in A$; then $U=a+A$ is a neighborhood of 0 and $U<a$. Since $a>0$ we have $U<n a$ for $n=1,2, \cdots$. If $\Xi$ is compact 0 must be a limit point of $\{n a\}_{1}^{\infty}$, and thus $n a \in U$ for some (in fact infinitely many) values of $n$, a contradiction.

LEMMA $2 \mathrm{~b}$. If $\Xi$ has a measurable order then $\Xi$ is locally $R_{n}$ for some $n \geqq 0$.

Proof. Here $R_{n}$ is $n$-dimensional Euclidean space considered as an additive group if $n \geqq 1$, while $R_{0}$ is the trivial group consisting of one element. By a basic structure theorem, see $[10$, p. 40$], \Xi$ has an open subgroup which is a direct sum of a compact group $\Xi_{0}$ and $R_{n}, n \geqq 0$. The measurable order on $\Xi$ induces a measurable order on $\Xi_{0}$. It now follows from Lemma $2 a$ that $\Xi_{0}$ is trivial.

A measurable order on $R_{n}$ can be constructed by choosing a basis $\eta_{1}, \cdots, \eta_{n}$ for $R_{n}$ considered as an $n$-dimensional vector space over the reals and defining

$$
a_{1} \eta_{1}+\cdots+a_{n} \eta_{n}<b_{1} \eta_{1}+\cdots+b_{n} \eta_{n}
$$

if for some $m, a_{m}<b_{m}$ but $a_{k}=b_{k}$ for $k=m+1, \cdots, n$. The following result asserts that such lexicograph orders are the only measurable orders on $R_{n}$.

THEOREM 2c. Let $R_{n}$ have a measurable order " $<$ ". Then there exists a basis $\eta_{1}, \cdots, \eta_{n}$ for $R_{n}$ such that the order on $R_{n}$ is the lexicographic order defined by (1) above.

Proof. Let us first consider $R_{1}$. We know that the set $\{\xi \mid \xi>0\}$ contains an open set $U$ and consequently $\{\xi \mid \xi>0\}$ contains $\bigcup r U$ where $r$ ranges over all positive rational numbers (positive in the usual sense). The assertion of our theorem is an easy consequence of this.

We assert that $R_{n}$ can be considered not only as an ordered group but as an ordered vector space. Suppose that $\xi>0$ in $R_{n}$ and let $S(\xi)$ be the one dimensional subspace of $R_{n}$ (considered as a vector space over the reals) generated by $\xi$. The measureable order " $<$ " on $R_{n}$ induces a measurable order on $S(\xi)$. By the 
above $\eta \in S(\xi)$ is positive if and only if $\eta=a \xi$ where $a$ is a positive real number (in the usual sense), which proves our assertion.

We now proceed by induction. Suppose our result is known up to $n-1$; we will show that it is true for $n$. Let $R_{n}^{+}$be the set of those elements $\xi$ of $R_{n}$ such that

$$
\bigcup_{m=1}^{\infty}\{\eta \mid \eta<m \xi\}=R_{n},
$$

and let $R_{n}^{-}$be the set of those $\xi$ such that

$$
\bigcup_{m=1}^{\infty}\{\eta \mid \eta>m \xi\}=R_{n} .
$$

We assert that $R_{n}^{+}$is not empty . Let $\xi_{1}, \cdots, \xi_{n}$ be a basis for $R_{n}$ considered as a vector space over the reals. Since if $\xi_{k}<0$ we can replace $\xi_{k}$ by $-\xi_{k}$ we can assume $\xi_{k}>0, k=1, \cdots, n$. We assert that $\xi_{0}=\xi_{1}+\xi_{2}+\cdots+\xi_{n}$ is in $R_{n}^{+}$. Given $\eta \in R_{n}$ let $\eta=a_{1} \xi_{1}+a_{2} \xi_{2}+\cdots+a_{n} \xi_{n}$ where $a_{1}, \cdots, a_{n}$ are real numbers. Choose a positive integer $m$ so that $m>a_{k}, k=1, \cdots, n$. Then

$$
m \xi_{0}-\eta=\left(m-a_{1}\right) \xi_{1}+\left(m-a_{2}\right) \xi_{2}+\cdots+\left(m-a_{n}\right) \xi_{n} .
$$

By the case $n=1$ of our theorem $\left(m-a_{k}\right) \xi_{k}>0, k=1, \cdots, n$ and thus $m \xi_{0}>\eta$. We further assert that $R_{n}^{+}$is open. Let $\xi \in R_{n}^{+}$. Choose $m$ so large that $m \xi \geqq \xi_{1}+\cdots+\xi_{n}$ and let

$$
U=\left\{a_{1} \xi_{1}+\cdots+a_{n} \xi_{n}|| a_{i} \mid<1 / 2 m, i=1, \cdots, n\right\} .
$$

Then $\xi>2 U$ or $\xi-U>U$. This implies that $\xi-U \in R_{n}^{+}$, but $\xi-U$ is a neighborhood of $\xi$. Similarly $R_{n}^{-}$is nonempty and open.

Let $R_{n}^{0}=R_{n}-\left\{R_{n}^{+} \cup R_{n}^{-}\right\}$. We assert that $R_{n}^{0}$ is a vector subspace of $R_{n}$. If $\xi_{1}, \xi_{2} \in R_{n}^{0}$ then by definition there exist $\eta_{1}$ and $\eta_{2}$ in $R_{n}$ such that $m \xi_{1}<\eta_{1}$ and $m \xi_{2}<\eta_{2}$ for all $m=1,2, \cdots$. But then $m\left(\xi_{1}+\xi_{2}\right)<\eta_{1}+\eta_{2}$ for all $m$ so that $\xi_{1}+\xi_{2} \notin R_{n}^{+}$. Similarly $\xi_{1}+\xi_{2} \notin R_{n}^{-}$and thus $\xi_{1}+\xi_{2} \in R_{n}^{0}$, etc.

We have $R_{n}=R_{n}^{+} \cup R_{n}^{-} \cup R_{n}^{0}$. If $\operatorname{dim} R_{n}^{0}<n-1, R_{n}^{0}$ cannot disconnect $R_{n}$ and therefore $\operatorname{dim} R_{n}^{0}=n-1$. By induction there exists a basis $\eta_{1}, \cdots, \eta_{n-1}$ in $R_{n}^{0}$ such that $a_{1} \eta_{1}+\cdots+a_{n-1} \eta_{n-1}<b_{1} \eta_{1}+\cdots+b_{n-1} \eta_{n-1}$ if and only if for some $m, a_{m}<b_{m}$ while $a_{k}=b_{k}$ for $k=m+1, \cdots, n-1$. Take for $\eta_{n}$ any element of $R_{n}^{+}$; then we assert that $a_{1} \eta_{1}+\cdots+a_{n} \eta_{n}<b_{1} \eta_{1}+\cdots+b_{n} \eta_{n}$ if and only if for some $m, a_{m}<b_{m}$ and $a_{k}=b_{k}$ for $k=m+1, \cdots, n$. We may suppose that $a_{n} \neq b_{n}$ for otherwise this assertion follows from our induction assumption. Suppose $a_{n}<b_{n}$. If $a_{1} \eta_{1}+\cdots+a_{n} \eta_{n}<b_{1} \eta_{1}+\cdots+b_{n} \eta_{n}$ does not hold, we would then have

$$
\left(a_{1}-b_{1}\right) \eta_{1}+\cdots+\left(a_{n-1}-b_{n-1}\right) \eta_{n-1}>\left(b_{n}-a_{n}\right) \eta_{n} .
$$

Now $\left(b_{n}-a_{n}\right) \eta_{n} \in R_{n}^{+}$. If $\xi_{1} \in R_{n}^{+}$and $\xi_{2}>\xi_{1}$ then clearly $\xi_{2} \in R_{n}^{+}$as well. However, $\left(a_{1}-b_{1}\right) \eta_{1}+\cdots+\left(a_{n-1}-b_{n-1}\right) \eta_{n-1} \in R_{n}^{0}$, a contradiction. The case $a>b_{n}$ can be treated similarly. Q.E.D. 
By a partition $\pi$ of $\Xi$ we mean a decomposition of $\Xi$ into a finite number of disjoint intervals. An interval is a set of the form

$$
\left\{\eta^{\prime}<\eta<\eta^{\prime \prime}\right\},\left\{\eta^{\prime} \leqq \eta<\eta^{\prime \prime}\right\},\left\{\eta^{\prime}<\eta \leqq \eta^{\prime \prime}\right\} \text { or }\left\{\eta^{\prime} \leqq \eta \leqq \eta^{\prime \prime}\right\} \text {. }
$$

Here $\eta^{\prime}$ is in $\Xi$ or $-\infty$ and $\eta^{\prime \prime}$ is in $\Xi$ or $+\infty$. Note that $-\infty \leqq \eta$ and $-\infty<\eta$ mean the same thing, etc. Also a single point is an interval. An example of a partition $\pi=\{\Delta(j)\}_{j=1}^{4}$ is:

$$
\begin{aligned}
& \Delta(1)=\left\{-\infty<\eta<\eta_{1}\right\}, \Delta(2)=\left\{\eta=\eta_{1}\right\}, \\
& \Delta(3)=\left\{\eta_{1}<\eta<\eta_{2}\right\}, \quad \Delta(4)=\left\{\eta_{2} \leqq \eta<\infty\right\},
\end{aligned}
$$

where $-\infty \neq \eta_{1}<\eta_{2} \neq \infty$. The final theorem of this section is the following technical result which is needed later.

THEOREM 2d. Let $\boldsymbol{h}(d \xi)$ be a bounded Radon measure on $\Xi$. Then given $\varepsilon>0$ there is a partition $\pi$ of $\Xi$ such that if $\pi$ consists of the intervals $\Delta(1), \Delta(2), \cdots, \Delta(m)$ we have

$$
\int_{\Delta(k)}|\boldsymbol{h}(d \xi)|<\varepsilon
$$

for all $k$ for which $\Delta(k)$ is not a point.

Proof. It is easy to see fromछTheorem $2 c$ that the result is true if $\Xi=R_{n}$. In the general case $\Xi$ is, by Lemma $2 b$, 'alunion of open and closed cosets of the form $R_{n}+\zeta, n \geqq 0$. If $C$ is any compact subset it is contained in a finite union of such cosets. Therefore there exist $\zeta_{1}, \cdots, \zeta_{r}$ such that if

$$
A=\bigcup_{k=1}^{r}\left[R_{n}+\zeta_{k}\right], \quad B=\Xi-A,
$$

then

$$
\int_{B}|\boldsymbol{h}(d \xi)|<\frac{\varepsilon}{2} .
$$

By our initial remark we can for each $k, k=1, \cdots, r$, find a partition $\pi^{*}(k)=\left\{\Delta^{*}(k, j)\right\}_{j}$ of $R_{n}+\zeta_{k}$ (note $\pi^{*}(k)$ is not a partition of $\left.\Xi\right)$ such that for all $j$ for which $\Delta^{*}(k, j)$ is not a point

$$
\int_{\Delta^{*}(k, j)}|\boldsymbol{h}(d \xi)|<\frac{\varepsilon}{2 r} .
$$

We extend $\pi^{*}(k)=\left\{\Delta^{*}(k, j)\right\}_{j}$ to a partition $\pi(k)$ of $\Xi$ as follows. If, for example,

$$
\Delta^{*}(k, j)=\left\{\eta \in R_{n}+\zeta_{k} \mid \eta^{\prime}<\eta \leqq \eta^{\prime \prime}\right\}
$$

where $\eta^{\prime}, \eta^{\prime \prime} \in R_{n}+\zeta_{k}$ we set

$$
\Delta(k, j)=\left\{\eta \in \Xi \mid \eta^{\prime}<\eta \leqq \eta^{\prime \prime}\right\},
$$


and take $\pi(k)=\left\{\Delta(k, j)\right.$, . Having done this we let $\pi=\{\Delta(j)\}_{j}$ be the "common refinement" of the $\pi(k), k=1, \cdots, r$. Note that for each $j$ and $k$ there exists $m(j, k)$ such that $\Delta(j) \cap\left[R_{n}+\zeta_{k} j \subset \Delta^{*}(k, m)\right.$ and also that if $\Delta^{*}(k, m)$ itself is a point, then $\Delta(j)$ reduces to this point. Suppose $\Delta(j)$ is not a point; then

$$
\begin{aligned}
\int_{\Delta(j)}|h(d \xi)| & =\sum_{k=1}^{r} \int_{\Delta(j) \cap\left[R_{n}+\zeta_{k}\right]}|h(d \xi)|+\int_{\Delta(j) \cap B}|h(d \xi)| \\
& <\sum_{k=1}^{r} \frac{\varepsilon}{2 r}+\frac{\varepsilon}{2}=\varepsilon .
\end{aligned}
$$

It seems overwhelmingly probable that none of the results of the present section are new; however, I have not been able to find references for them.

3. Basic definitions. Let $\mathscr{M}_{0}(\Xi)$ be the Banach algebra of all bounded Radon measures on $\Xi$ with norm

$$
\|f(d \xi)\|_{0}=\int_{\Xi}|f(d \xi)|,
$$

and with convolution as multiplication. Let $\mathscr{M}_{0}(\Theta)$ be the Banach algebra of all Fourier-Stieltjes transforms of measures in $\mathscr{M}_{0}(\Xi)$,

$$
f(\theta)=\int_{\Xi}(\xi, \theta) f(d \xi),
$$

with norm

$$
\|f\|_{0}=\|f\|_{0},
$$

and with ordinary multiplication. The mapping $\mathscr{F}: f \rightarrow f$ is an isometric isomorphism of $\mathscr{M}_{0}(\Xi)$ onto $\mathscr{M}_{0}(\Theta)$.

For fixed $\eta \in \Xi$ let the functions on the left below be the characteristic functions of the sets on the right:

$$
\begin{aligned}
\chi^{+}(\eta, \xi) & \leftrightarrow\{\xi \mid \xi \geqq \eta\} ; \\
\chi^{+}(\eta+, \xi) & \leftrightarrow\{\xi \mid \xi>\eta\} ; \\
\chi^{-}(\eta, \xi) & \leftrightarrow\{\xi \mid \xi \leqq \eta\} ; \\
\chi^{-}(\eta-, \xi) & \leftrightarrow\{\xi \mid \xi<\eta\} .
\end{aligned}
$$

We define

$$
\begin{aligned}
\boldsymbol{E}^{+}(\eta) \boldsymbol{f} \cdot(d \xi) & =\chi^{+}(\eta, \xi) \boldsymbol{f}(d \xi), \\
\boldsymbol{E}^{+}(\eta+) \boldsymbol{f} \cdot(d \xi) & =\chi^{+}(\eta+, \xi) \boldsymbol{f}(d \xi), \\
\boldsymbol{E}^{-}(\eta) \boldsymbol{f} \cdot(d \xi) & =\chi^{-}(\eta, \xi) \boldsymbol{f}(d \xi), \\
\boldsymbol{E}^{-}(\eta-) \boldsymbol{f} \cdot(d \xi) & =\chi^{-}(\eta-, \xi) \boldsymbol{f}(d \xi) .
\end{aligned}
$$


It is apparent that $E^{+}(\eta), E^{+}(\eta+)$, etc. are all projections of norm 1 on $\mathscr{M}_{0}(\Xi)$ considered as a Banach space.

Let us write

$$
\boldsymbol{f}(d \xi)<<\boldsymbol{g}(d \xi)
$$

if

$$
\int_{B}|f(d \xi)| \leqq \int_{B}|g(d \xi)|
$$

for every Borel set $B$ in $\Xi$.

Definition 3a. Let $\mathscr{M}(\Xi)$ be a subalgebra of $\mathscr{M}_{0}(\Xi)$ with unit and let $\|\cdot\|$ be a norm on $\mathscr{M}(\Xi)$ such that $\{\mathscr{M}(\Xi),\|\cdot\|\}$ is a Banach algebra. $\mathscr{M}(\Xi)$ will be said to be of type $\mathfrak{M}$ if :

(i) for every $\eta \in \Xi$ the operators $\boldsymbol{E}^{+}(\eta), \boldsymbol{E}^{+}(\eta+), \boldsymbol{E}^{-}(\eta), \boldsymbol{E}^{-}(\eta-)$ restricted to $\mathscr{M}(\Xi)$ map $\mathscr{M}(\Xi)$ into itself and have norm 1 ;

(ii) for every $f \in \mathscr{M}(\Xi)$

$$
\begin{aligned}
& \lim _{\eta \rightarrow+\infty}\left\|\boldsymbol{E}^{+}(\eta) \boldsymbol{f}\right\|=\lim _{\eta \rightarrow+\infty}\left\|\boldsymbol{E}^{+}(\eta+) \boldsymbol{f}\right\|=0, \\
& \lim _{\eta \rightarrow-\infty}\left\|\boldsymbol{E}^{-}(\eta) \boldsymbol{f}\right\|=\lim _{\eta \rightarrow-\infty}\left\|\boldsymbol{E}^{-}(\eta-) \boldsymbol{f}\right\|=0 .
\end{aligned}
$$

If in addition the following condition holds $\mathscr{M}(\Xi)$ will be said to be of type $\Im$ : (iii) $\boldsymbol{f}<<\boldsymbol{g}, \boldsymbol{g} \in \mathscr{M}(\Xi)$ implies that $\boldsymbol{f} \in \mathscr{M}(\Xi)$ and $\|f\| \leqq\|g\|$.

Note that the definition of type $\mathfrak{S}$ given here is weaker than the definition given in [9].

Let $\mathscr{M}(\Theta)$ be the set of Fourier-Stieltjes transforms of measures in $\mathscr{M}(\Xi)$ and set $\|f\|=\|\boldsymbol{f}\|$. Then $\mathscr{M}(\Theta)$ is a Banach algebra of functions and the mapping $\mathscr{F}: f \rightarrow f$ is an isometric isomorphism of $\mathscr{M}(\Xi)$ onto $\mathscr{M}(\Theta)$. We say that $\mathscr{M}(\Theta)$ is of type $\mathfrak{M}(\Im)$ if $\mathscr{M}(\Xi)$ is of type $\mathfrak{M}(\mathfrak{\Xi})$.

We further define

$$
\begin{gathered}
\boldsymbol{E}^{+}(\eta) f=\mathscr{F} \boldsymbol{E}^{+}(\eta) \mathscr{F}^{-1} f, \\
\boldsymbol{E}^{+}(\eta+) f=\mathscr{F} \boldsymbol{E}^{+}(\eta+) \mathscr{F}^{-1} f,
\end{gathered}
$$

etc.

Let $\mathscr{M}(\Theta)$ be of type $\mathfrak{S}$. We will write

$$
f \prec g, \quad f, g \in \mathscr{M}(\Theta)
$$

if

$$
\boldsymbol{f}<<\boldsymbol{g}
$$

where $\boldsymbol{f}=\mathscr{F}^{-1} f, \boldsymbol{g}=\mathscr{F}^{-1} g$. We also define 


$$
f^{*}(\theta)=\int_{\Xi}(\xi, \theta)|f(d \xi)| \text {. }
$$

It is apparent that $f^{\#} \in \mathscr{M}(\Theta)$ with $f$ and that $\left\|f^{\#}\right\|=\|f\|$.

Let $\xi_{1}, \xi_{2} \in \Xi$; we will write $f(d \xi) \in\left(\xi_{1}, \xi_{2}\right]$ if $B \cap\left\{\xi \mid \xi_{1}<\xi \leqq \xi_{2}\right\}=\varnothing$ implies that $\int_{B}|f(d \xi)|=0$ for every $B \in \mathscr{B}$. Similarly we will write $f \in\left(\xi_{1}, \xi_{2}\right]$ if $f \in\left(\xi_{1}, \xi_{2}\right]$ where (as usual) $\boldsymbol{f}=\mathscr{F}^{-1} f$, etc.

4. Wiener-Hopf equations. Suppose that $\mathscr{M}(\Theta)$ is of type $\mathfrak{M}$. For $c \in \mathscr{M}(\Theta)$ we define the Wiener-Hopf operators

$$
\begin{array}{ll}
W_{c}^{+} f=E^{+}(0) c f, & f \in E^{+}(0) \mathscr{M}(\Theta), \\
W_{c}^{-} f=E^{-}(0) c f, & f \in E^{-}(0) \mathscr{M}(\Theta) .
\end{array}
$$

Clearly $W_{c}^{+}$is a bounded linear mapping of $E^{+}(0) M(\Theta)$ (considered as a Banach space) into itself and $\left\|W_{c}^{+}\right\| \leqq\|c\|$, etc. We will write $c \in W H[\mathscr{M}(\Theta)]$ if both $W_{c}^{+}$and $W_{c}^{-}$have (bounded) inverses.

THEOREM 4a. $c \in W H[\mathscr{M}(\Theta)]$ if and only if

$$
c=d^{2} U^{-1} V^{-1}
$$

where $d$ is a nonzero constant, $U, U^{-1} \in E^{+}(0) \mathscr{M}(\Theta)$, and $V, V^{-1} \in E^{-}(0) \mathscr{M}(\Theta)$.

Proof. By assumption there are functions $U(\theta) \in E^{+}(0) \mathscr{M}(\Theta)$ and $V(\theta) \in E^{-}(0) \mathscr{M}(\Theta)$ such that

$$
W_{c}^{+} U=1, \quad W_{c}^{-} V=1 .
$$

For future use we introduce the notation

$$
\begin{aligned}
& U(\theta)=\int_{\xi \geqq 0}(\xi, \theta) U(d \xi), \\
& V(\theta)=\int_{\xi \leqq 0}(\xi, \theta) V(d \xi) .
\end{aligned}
$$

Let

$$
V_{1}(\theta)=c(\theta) U(\theta), U_{1}(\theta)=c(\theta) V(\theta) .
$$

Then $U_{1}, V_{1} \in \mathscr{M}(\Theta)$, and

$$
\begin{array}{ll}
V_{1}(\theta)=\int_{\xi \leqq 0}(\xi, \theta) V_{1}(d \xi), & V_{1}\{(0\})=1, \\
U_{1}(\theta)=\int_{\xi \geqq 0}(\xi, \theta) U_{1}(d \xi), & U_{1}(\{0\})=1 .
\end{array}
$$


By definition

$$
\begin{gathered}
V(\theta) V_{1}(\theta)=c(\theta) U(\theta) V(\theta)=U(\theta) U_{1}(\theta), \\
V * V_{1}=U * U_{1} .
\end{gathered}
$$

Now $V * V_{1}$ has support in $\{\xi \leqq 0\}$ and $U * U_{1}$ has support in $\{\xi \geqq 0\}$. It follows that $V * V_{1}$ and $U * U_{1}$ both have support in $\{0\}$. Thus there is a complex constant $d$ such that

$$
V(\theta) V_{1}(\theta)=d^{2}=U(\theta) U_{1}(\theta)
$$

We have

$$
c(\theta) U(\theta) \overline{U_{1}(\theta)}=V_{1}(\theta) \overline{U_{1}(\theta)} .
$$

Let $\bar{U}_{1}=\mathscr{F}^{-1} \bar{V}_{1}$. Then $\bar{U}_{1}$ has support in $\{\xi \leqq 0\}$ and

$$
V_{1} * \bar{U}_{1} \cdot(\{0\})=1
$$

Thus $V_{1}(\theta) \overline{U_{1}(\theta)} \not \equiv 0$ from which it follows that $U(\theta) \overline{U_{1}(\theta)} \not \equiv 0, U(\theta) U_{1}(\theta) \not \equiv 0$, and $d \neq 0$. Since $U(\theta)^{-1}=d^{-2} U_{1}(\theta)$ we have $U(\theta)^{-1} \in E^{+}(0) \mathscr{M}(\Theta)$, and similarly $V(\theta)^{-1} \in E^{-}(0) \mathscr{M}(\Theta)$. Thus our conditions are necessary.

Suppose now that (2) holds. We define operators $X_{c}^{+}$and $X_{c}^{-}$on $E^{+}(0) \mathscr{M}(\Theta)$, and $E^{-}(0) \mathscr{M}(\Theta)$ respectively by the formulas

$$
\begin{array}{ll}
X_{c}^{+} f \cdot(\theta)=d^{-2} U(\theta) E^{+}(0) f(\theta) V(\theta), & f \in E^{+}(0) \mathscr{M}(\Theta), \\
X_{c}^{-} f \cdot(\theta)=d^{-2} V(\theta) E^{-}(0) f(\theta) U(\theta), & f \in E^{-}(0) \mathscr{M}(\Theta) .
\end{array}
$$

It is not difficult to verify that

$$
\begin{aligned}
& X_{c}^{+} W_{c}^{+} f=W_{c}^{+} X_{c}^{+} f=f \text { for } f \in E^{+}(0) \mathscr{M}(\Theta), \\
& X_{c}^{-} W_{c}^{-} f=W_{c}^{-} X_{c}^{-} f=f \text { for } f \in E^{-}(0) \mathscr{M}(\Theta) .
\end{aligned}
$$

See $[9, \S 3]$.

We further note that it follows from (3) that

$$
U(\{0\})=V(\{0\})=d^{2} .
$$

The result given above is closely related to one due to Krein, Uspehi Mat. Nauk 13 (83) (1958), 3-120, and Calderon, Spitzer, and Widom, Illinois J. Math. 3 (1959), 490-497.

It is clear from the above that if $\mathscr{M}(\Theta)$ is of type $\mathfrak{M}$ then

$$
c \in W H[\mathscr{M}(\Theta)] \Rightarrow c \in W H\left[\mathscr{M}_{0}(\Theta)\right],
$$

a fact which we will have occasion to use later. 
5. Finite section Wiener-Hopf operators $\left({ }^{1}\right)$. For $\eta \geqq 0$ let

$$
\begin{array}{ll}
W_{c}^{+}(\eta) f=E^{-}(\eta) E^{+}(0) c f, & f \in E^{-}(\eta) E^{+}(0) \mathscr{M}(\Theta), \\
W_{c}^{-}(\eta) f=E^{+}(-\eta) E^{-}(0) c f, & f \in E^{+}(-\eta) E^{-}(0) \mathscr{M}(\Theta) .
\end{array}
$$

$W_{c}^{+}(\eta)$ and $W_{c}^{-}(\eta)$ are bounded linear operators on the Banach spaces $E^{-}(\eta) E^{+}(0) \mathscr{M}(\Theta)$ and $E^{+}(-\eta) E^{-}(0) \mathscr{M}(\Theta)$ respectively. Baxter [3] showed in the case $\Xi=I$ and $\Theta=T$ ( $I$ is the integers and $T$ the reals modulo 1 ) that if $c \in W H[\mathscr{M}(\Theta)]$, where $\mathscr{M}(\Theta)$ is a Beurling-Gelfand subalgebra of $\mathscr{M}_{0}(\Theta)$, then $\left(W_{c}^{+}(\eta)\right)^{-1}$ and $\left(W_{c}^{-1}(\eta)\right)^{-1}$ also exist for all sufficiently large $\eta, \eta \geqq \zeta_{1}$. In the present section we will prove a more general result of this kind. Our demonstration is based on the following identity due to Shinbrot [11]. See also [4] and [9].

Let us for the moment set

$$
\begin{aligned}
& W_{c}^{+}(\eta) f=E^{+}(0) E^{-}(\eta) c E^{+}(0) E^{-}(\eta) f, \\
& W_{c}^{-}(\eta) f=E^{-}(0) E^{+}(-\eta) c E^{-}(0) E^{+}(-\eta) f,
\end{aligned}
$$

and regard $W_{c}^{+}(\eta)$ as defined on $\mathscr{M}(\Theta)$ with range in $E^{+}(0) E^{-}(\eta) \mathscr{M}(\Theta)$, etc.

THEOREM 5a. Let $\mathscr{M}(\Theta)$ be of type $\mathfrak{M}$, and let $c(\theta)=u(\theta)^{-1} v(\theta)^{-1}$, where $u(\theta) \in E^{+}(0) E^{-}(\zeta) \mathscr{M}(\Theta), \quad u(\theta)^{-1} \in^{\top} E^{+}(0) \mathscr{M}(\Theta), \quad v(\theta) \in E^{-}(0) E^{+}(-\zeta) \mathscr{M}(\Theta)$, $v(\theta)^{-1} \in E^{-}(0) \mathscr{M}(\Theta)$. We set

$$
\begin{aligned}
& Y_{c}^{+}(\eta) f=v E^{-}(\eta) v^{-1} u E^{+}(0) v f \\
& Y_{c}^{-}(\eta) f=u E^{+}(-\eta) u^{-1} v E^{-}(0) u f .
\end{aligned}
$$

Then for $\eta \geqq \zeta \geqq 0$ we have

$$
\begin{aligned}
& Y_{c}^{+}(\eta) W_{c}^{+}(\eta)=W_{c}^{+}(\eta) Y_{c}^{+}(\eta)=E^{+}(0) E^{-}(\eta), \\
& Y_{c}^{-}(\eta) W_{c}^{-}(\eta)=W_{c}^{-}(\eta) Y_{c}^{-}(\eta)=E^{-}(0) E^{+}(-\eta) .
\end{aligned}
$$

From this point on we return to our original point of view of considering $W_{c}^{+}(\eta)$ as a transformation of $E^{+}(0) E^{-}(\eta) \mathscr{M}(\Theta)$ into itself, etc.

Using Theorem $5 \mathrm{a}$ we can prove the following basic result. Since the demonstration is almost word for word like that given in [9] it is omitted.

THEOREM 5b. Let $\mathscr{M}(\Theta)$ be of type $\mathfrak{M}$. If $c \in W H[\mathscr{M}(\Theta)]$ there exists $\zeta_{1} \geqq 0$ in $\Xi$ and a positive constant $A$ such that if $\eta \geqq \zeta_{1}$ we have:

a. $\|f\| \leqq\left\|W_{c}^{+}(\eta) f\right\|$ if $f \in E^{-}(\eta) E^{+}(0) \mathscr{M}(\Theta)$ and the range of $W_{c}^{+}(\eta)$ is all of $E^{-}(\eta) E^{+}(0) \mathscr{M}(\Theta)$;

b. $\|f\| \leqq A\left\|W_{c}^{-}(\eta) f\right\|$ if $f \in E^{+}(-\eta) E^{-}(0) \mathscr{M}(\Theta)$ and the range of $W_{c}^{-}(\eta)$ is all of $E^{+}(-\eta) E^{-}(0) \mathscr{M}(\Theta)$.

(1) We use "finite section Wiener-Hopf operators" rather than "Toeplitz operators". 
If in addition $\mathscr{M}(\Theta)$ is of type $\subseteq$ then there exist $C^{+}$and $C^{-}$in $\mathscr{M}(\Theta)$ with $C^{+}=\left(C^{+}\right)^{*}, C^{-}=\left(C^{-}\right)^{*}$, such that if $\eta \geqq \zeta_{1}$ we have

c. $f \prec C^{+}\left[W_{c}^{+}(\eta) f\right]^{*}, f \in E^{-}(\eta) E^{+}(0) \mathscr{M}(\Theta)$,

$$
f \prec C^{-}\left[W_{c}^{-}(\eta) f\right]^{*}, f \in E^{+}(-\eta) E^{+}(0) \mathscr{M}(\Theta) .
$$

Corollary 5c. Let $\mathscr{M}(\Theta)$ be of type $\mathfrak{M}$. Let $c(\theta) \in W H[\mathscr{M}(\Theta)]$ and let $g(\theta) \in E^{+}(0) \mathscr{M}(\Theta)$. For each $\eta \geqq \zeta_{1}$ let

$$
\begin{aligned}
f(\eta, \theta) & =\left\{W_{c}^{+}(\eta)\right\}^{-1} E^{-}(\eta) g(\theta), \\
f(\theta) & =\left\{W_{c}^{+}\right\}^{-1} g(\theta) .
\end{aligned}
$$

Then there is a constant $A$ independent of $g$ such that

$$
\|f(\theta)-f(\eta, \theta)\| \leqq A\left\|E^{+}(\eta+) f\right\| .
$$

If in addition $\mathscr{M}(\Theta)$ is of type $\subseteq$ then

$$
f(\theta)-f(\eta, \theta) \prec 2 C^{+}(\theta) E^{+}(\eta+)\left[c^{*}(\theta) C^{+}(\theta) g^{*}(\theta)\right] .
$$

Proof. For the proof of results similar to (1) see [3] and [9]. To prove (2) we note that by $\mathrm{c}$ of Theorem $5 \mathrm{~b}$

$$
f(\eta, \cdot) \prec C^{+} E^{-}(\eta) g^{\#} \prec C^{\#} g^{\#} .
$$

Clearly this holds for " $\eta=\infty$ " as well so that

$$
f \prec C^{+} g^{\#} \text {. }
$$

Since $W_{c}^{+} f(\cdot)$ and $W_{c}^{+} f(\eta, \cdot)$ agree on $[0, \eta]$ we have

$$
W_{c}^{+}[f(\cdot)-f(\eta, \cdot)]=E^{+}(\eta+)[c f(\cdot)+c f(\eta, \cdot)]
$$

from which it follows that

$$
W_{c}^{+}[f(\cdot)-f(\eta \cdot)] \prec 2 E^{+}(\eta+) c^{\#} C^{+} g^{\#},
$$

and hence, using (3) again, that

$$
f(\theta)-f(\eta, \theta) \prec 2 C^{+}\left[E^{+}(\eta+) c^{\#} C^{+} g^{\#}\right] .
$$

There is, of course, a similar result associated with $W_{c}^{-}$. We will regard Corollary $5 \mathrm{~b}$ as referring to this case as well.

Let $\mathscr{M}(\Theta)$ be of type $\mathfrak{M}$ and let $c \in W H[\mathscr{M}(\Theta)]$. For $\eta \geqq \zeta_{1}$ we define $u(\eta, \theta)$ and $v(\eta, \theta)$ by

$$
\begin{array}{ll}
W_{c}^{+}(\eta) u(\eta, \theta)=1, & u(\eta, \theta) \in E^{-}(\eta) E^{+}(0) \mathscr{M}(\Theta), \\
W_{c}^{-}(\eta) v(\eta, \theta)=1, & v(\eta, \theta) \in E^{+}(-\eta) E^{-}(0) \mathscr{M}(\Theta) .
\end{array}
$$

These functions play a fundamental role in all that follows. By Corollary $5 \mathrm{c}$ 


$$
\lim _{\eta}\|U(\theta)-u(\eta, \theta)\|=\lim _{\eta}\|V(\theta)-v(\eta, \theta)\|=0
$$

Since $U(\theta)$ has an inverse $U(\theta)^{-1}$ in the Banach algebra $E^{+}(0) \mathscr{M}(\Theta)$ and since $V(\theta)$ has an inverse $V(\theta)^{-1}$ in the Banach algebra $E^{-}(0) \mathscr{M}(\Theta)$ it follows that there exists $\zeta_{1}^{\prime} \geqq \zeta_{1}$ such that

$$
\begin{aligned}
& u(\eta, \theta)^{-1} \in E^{+}(0) \mathscr{M}(\Theta), \\
& v(\eta, \theta)^{-1} \in E^{-}(0) \mathscr{M}(\Theta),
\end{aligned}
$$

See Hille and Phillips, Functional analysis and semi-groups, Amer. Math. Soc. Colloq. Publ. Vol. 31, rev. ed., Providence, R. I., 1957, p. 118. We assert that for $\eta>\zeta_{1}$

$$
u(\eta,\{0\})=v(\eta,\{0\})
$$

This follows from the fact that, as is easily verified,

$$
\begin{aligned}
& {[(c(\cdot) * u(\eta, \cdot)) * v(\eta, \cdot)] \cdot(\{0\})=v(\eta,\{0\}),} \\
& {[(c(\cdot) * v(\eta, \cdot)) * u(\eta, \cdot)] \cdot(\{0\})=u(\eta,\{0\})}
\end{aligned}
$$

We can therefore set

$$
d(\eta)^{2}=u(\eta,\{0\})=v(\eta,\{0\}), \quad \eta \geqq \zeta_{1} .
$$

It follows easily from the fact that $v(\eta, \theta)^{-1} \in E^{-}(0) \mathscr{M}(\Theta)$ that $d(\eta) \neq 0$ for $\eta \geqq \zeta_{1}^{\prime}$. For $\eta \geqq \zeta_{1}^{\prime}$ we define

$$
c_{\eta}(\theta)=\frac{d(\eta)^{2}}{u(\eta, \theta) v(\eta, \theta)}
$$

THEOREM 5d. Under the above assumptions if $\eta \geqq \zeta_{1}^{\prime}$ then

$$
c_{\eta}(B)=c(B)
$$

for every Borel set $B,-\eta \leqq B \leqq \eta$.

The proof of this theorem will follow from the following result which is of independent interest.

TheOREM 5e. Let $u \in E^{-}(\eta) E^{+}(0) \mathscr{M}_{0}(\Theta), u^{-1} \in E^{+}(0) \mathscr{M}_{0}(\Theta)$,

$$
v \in E^{+}(-\eta) E^{-}(0) \mathscr{M}_{0}(\Theta), \quad v^{-1} \in E^{-}(0) \mathscr{M}_{0}(\Theta) .
$$

We define linear operators $Q, R$ on $E^{+}(-\eta) E^{-}(\eta) \mathscr{M}_{0}(\Theta)$ as follows:

$$
\begin{aligned}
& Q f=u E^{-}(0-) f+v E^{+}(0) f \\
& R f=E^{-}(\eta) u E^{+}(0) u^{-1} v^{-1} f+E^{+}(-\eta) v E^{-}(0-) u^{-1} v^{-1} f .
\end{aligned}
$$

We assert that for $f \in E^{+}(-\eta) E^{-}(\eta) \mathscr{M}_{0}(\Theta)$

$$
Q R f=R Q f=f .
$$


Proof. We have $Q R f=I_{1}+I_{2}+I_{3}+I_{4}$ where

$$
\begin{aligned}
& I_{1}=u E^{-}(0-) E^{-}(\eta) u E^{+}(0) u^{-1} v^{-1} f, \\
& I_{2}=u E^{-}(0-) E^{+}(-\eta) v E^{-}(0-) u^{-1} v^{-1} f, \\
& I_{3}=v E^{+}(0) E^{-}(\eta) u E^{+}(0) u^{-1} v^{-1} f \\
& I_{4}=v E^{+}(0) E^{+}(-\eta) v E^{-}(0-) u^{-1} v^{-1} f .
\end{aligned}
$$

Since $E^{-}(\eta) u E^{+}(0) u^{-1} v^{-1} f \in[0, \eta]$ it follows that $I_{1}=0$ and that

$$
I_{3}=v E^{-}(\eta) u E^{+}(0) u^{-1} v^{-1} f .
$$

Similarly since $E^{+}(-\eta) v E^{-}(0-) u^{-1} v^{-1} f \in[-\eta, 0)$ it follows that $I_{4}=0$ and that

We have

$$
I_{2}=u E^{+}(-\eta) v E^{-}(0-) u^{-1} v^{-1} f
$$

$$
\begin{aligned}
& I_{2}=u v E^{-}(0-) u^{-1} v^{-1} f-u E^{-}(-\eta-) v E^{-}(0-) u^{-1} v^{-1} f \\
& I_{3}=u v E^{+}(0) u^{-1} v^{-1} f-v E^{+}(\eta+) u E^{+}(0) u^{-1} v^{-1} f .
\end{aligned}
$$

Now

$$
\begin{aligned}
u E^{-}(-\eta-) v E^{-}(0-) u^{-1} v^{-1} f & =u E^{-}(\eta-) u^{-1} f-u E^{-}(\eta-) v E^{+}(0) u^{-1} v^{-1} f \\
& =0-0
\end{aligned}
$$

and

$$
\begin{aligned}
v E^{+}(\eta+) u E^{+}(0) u^{-1} v^{-1} f & =v E^{+}(\eta+) v^{-1} f-v E^{+}(\eta+) u E^{-}(0-) u^{-1} v^{-1} f \\
& =0-0 .
\end{aligned}
$$

Thus

$$
Q R f=u v E^{-}(0-) u^{-1} v^{-1} f+u v E^{+}(0) u^{-1} v^{-1} f=f .
$$

On the other hand $R Q f=J_{1}+J_{2}+J_{3}+J_{4}$ where

$$
\begin{aligned}
& J_{1}=E^{-}(\eta) u E^{+}(0) v^{-1} E^{-}(0-) f, \\
& J_{2}=E^{-}(\eta) u E^{+}(0) u^{-1} E^{+}(0) f, \\
& J_{3}=E^{+}(-\eta) v E^{-}(0-) v^{-1} E^{-}(0-) f, \\
& J_{4}=E^{+}(-\eta) v E^{-}(0-) u^{-1} E^{+}(0) f .
\end{aligned}
$$

It is immediately apparent that $J_{1}=J_{4}=0$ and that $J_{2}=E^{-}(\eta) E^{+}(0) f$, $J_{3}=E^{+}(-\eta) E^{-}(0-) f$, so that

$$
Q R f=E^{-}(\eta) E^{+}(0) f+E^{+}(-\eta) E^{-}(0-) f=f
$$


For future use we set

$$
\boldsymbol{Q}=\mathscr{F}^{-1} Q \mathscr{F}, \boldsymbol{R}=\mathscr{F}^{-1} \boldsymbol{R} \mathscr{F} .
$$

We now return to the proof of Theorem $5 \mathrm{~d}$. In what follows $\alpha * \beta$ is a measure if $\alpha$ and $\beta$ are measures and $\alpha * f$ is a function if $\alpha$ is a measure and $f$ is a function. Let $f(\xi)$ be a bounded Borel measurable function vanishing except for $-\eta \leqq \xi \leqq \eta$, and let $\gamma(d \xi)=c(d \xi)-c_{\eta}(d \xi)$. Then $\{\gamma(\cdot) * u(\eta, \cdot)\}(d \xi)$ has no mass in $[0, \eta]$ and $\{\gamma(\cdot) * v(\eta, \cdot)\}(d \xi)$ has no mass in $[-\eta, 0]$. Thus if $\phi(\xi)^{\vee}=\phi(-\xi)$ then

and

$$
I^{\prime}=\int\left\{\boldsymbol{E}^{-}(0-) f(\xi)\right\}^{\vee}\{\boldsymbol{u}(\eta, \cdot) * \gamma(\cdot)\}(d \xi)=0
$$

We have

$$
I^{\prime \prime}=\int\left\{E^{+}(0) f(\xi)\right\}^{\vee}\{v(\eta, \cdot) * \gamma(\cdot)\}(d \xi)=0 .
$$

$$
\begin{aligned}
I^{\prime} & =\int\left\{\boldsymbol{u}(\eta, \cdot) * \boldsymbol{E}^{-}(0-) \boldsymbol{f} \cdot(\xi)\right\}^{\vee} \gamma(d \xi), \\
I^{\prime \prime} & =\int\left\{\boldsymbol{v}(\eta, \cdot) * \boldsymbol{E}^{+}(0) \boldsymbol{f} \cdot(\xi)\right\}^{\vee} \gamma(d \xi),
\end{aligned}
$$

from which it follows that

$$
\int\left\{u(\eta, \cdot) * E^{-}(0-) f \cdot(\xi)+v(\eta, \cdot) * E^{+}(0) f \cdot(\xi)\right\}^{\vee} \gamma(d \xi)=0
$$

that is

$$
\int\{\boldsymbol{Q f}(\xi)\}^{\vee} \gamma(d \xi)=0
$$

Let $g(\xi)$ be an arbitrary bounded Borel measurable function vanishing except for $-\eta \leqq \xi \leqq \eta$. It is apparent from the formula for $\boldsymbol{R}$ that if $\boldsymbol{f}=\boldsymbol{R g}, \boldsymbol{f}$ is a bounded Borel measurable function vanishing except for $-\eta \leqq \xi \leqq \eta$, and $Q f=g$. Thus

$$
0=\int\{\boldsymbol{Q R} \boldsymbol{g}\}^{\vee} \gamma(d \xi)=\int\{\boldsymbol{g}(\xi)\}^{\vee} \gamma(d \xi) .
$$

Since $g$ is arbitrary our theorem is proved. For $\Theta=T$ this result was proved by Baxter in [1], using a different argument.

As a consequence of Theorem $5 \mathrm{~d}$ we have the following explicit inverses for $W_{c}^{+}(\eta)$ and $W_{c}^{-}(\eta)$.

THEOREM 5f. Let $\mathscr{M}(\Theta)$ be of type $\mathfrak{M}$ and let $c \in W H[\mathscr{M}(\Theta)]$. Then there exists $\zeta \geqq 0$ so large that if $\eta \geqq \zeta$

Here

$$
\begin{aligned}
& Y_{c}^{+}(\eta) W_{c}^{+}(\eta)=W_{c}^{+}(\eta) Y_{c}^{+}(\eta)=I^{+}(\eta), \\
& Y_{c}^{-}(\eta) W_{c}^{-}(\eta)=W_{c}^{-}(\eta) Y_{c}^{-}(\eta)=I^{-}(\eta) .
\end{aligned}
$$

$$
Y_{c}^{+}(\eta) f(\theta)=d(\eta)^{-2} v(\eta, \theta) E^{-}(\eta) u(\eta, \theta) v(\eta, \theta)^{-1} E^{+}(0) v(\eta, \theta) f(\theta) .
$$


for $f \in E^{+}(0) E^{-}(\eta) \mathscr{M}(\Theta)$, and $I^{+}(\eta)$ is the identity operator on this space. Similarly

$$
Y_{c}^{-}(\eta) f \cdot(\theta)=d(\eta)^{-2} u(\eta, \theta) E^{+}(-\eta) u(\eta, \theta)^{-1} v(\eta, \theta) E^{-}(0) u(\eta, \theta) f(\theta)
$$

for $f \in E^{-}(0) E^{+}(-\eta) \mathscr{M}(\Theta)$ and $I^{-}(\eta)$ is the identity operator on this space.

This follows from Theorem 5a combined with the fact that, as a consequence of Theorem 5d,

$$
W_{c}^{+}(\eta) \equiv W_{c_{\eta}}^{+}(\eta), \quad W_{c}^{-}(\eta) \equiv W_{c_{\eta}}^{-}(\eta) .
$$

6. Representations of measures. Let $c(\theta) \in W H\left[\mathscr{M}_{0}(\Theta)\right]$, let $\zeta_{1}$ be as in Theorem 5a, etc. If $\eta>\zeta_{1}$ is fixed then $u(\eta, \theta)$ and $v(\eta, \theta)$ are defined and

$$
u(\eta, \theta) \prec C^{+}(\theta), \quad v(\eta, \theta) \prec C^{-}(\theta) .
$$

We can therefore apply the Radon-Nikodym theorem to deduce that there exist Borel measurable functions of $\xi, \boldsymbol{u}_{*}(\eta, \xi)$ and $\boldsymbol{v}_{*}(\eta, \xi)$ such that

$$
\begin{aligned}
& \boldsymbol{u}(\eta, d \xi)=\boldsymbol{u}_{*}(\eta, \xi) \boldsymbol{C}^{+}(d \xi), \\
& \boldsymbol{v}(\eta, d \xi)=\boldsymbol{v}_{*}(\eta, \xi) \boldsymbol{C}^{-}(d \xi) .
\end{aligned}
$$

We may further suppose that $\boldsymbol{u}_{*}(\eta, \xi)$ is 0 if $\xi$ is not in the support of $\boldsymbol{C}^{+}$, that $\left|\boldsymbol{u}_{*}(\eta, \xi)\right| \leqq 1$, and that $\boldsymbol{u}_{*}(\eta, \xi)=0$ except when $0 \leqq \xi \leqq \eta$. Similar remarks apply to $\boldsymbol{v}_{*}(\eta, \xi)$. The principal result of this section is that we can "make" $u_{*}(\eta, \xi)$ and $v_{*}(\eta, \xi)$ measurable in both $\xi$ and $\eta$.

THeOREM 6a. Let $c \in W H\left[\mathscr{M}_{0}(\Theta)\right]$ and let $\zeta_{1}$ be as in Theorem 5a. Given $\varepsilon>0$ there exists a partition of $\left(\zeta_{1}<\eta<\infty\right), \pi=\{\Delta(j)\}_{j}$, such that

$$
\left\|u\left(\eta_{1}, \theta\right)-u\left(\eta_{2}, \theta\right)\right\|_{0}<\varepsilon
$$

whenever $\eta_{1}$ and $\eta_{2}$ belong to the same interval $\Delta(j)$ of $\pi$.

Proof. Suppose $\zeta_{1}<\eta_{1}<\eta_{2}$. Then since

$$
c(\theta) u(\eta, \theta) \prec c^{\#} C^{+}
$$

and since

$$
\begin{aligned}
& c(\theta) u\left(\eta_{1}, \theta\right)=(-\infty, 0)+1+\left(\eta_{1}, \infty\right), \\
& c(\theta) u\left(\eta_{2}, \theta\right)=(-\infty, 0)+1+\left(\eta_{2}, \infty\right),
\end{aligned}
$$

it follows that

$$
W_{c}^{+}\left(\eta_{2}\right)\left[u\left(\eta_{1}, \cdot\right)-u\left(\eta_{2}, \cdot\right)\right] \prec E^{+}\left(\eta_{1}+\right) E^{-}\left(\eta_{2}\right) c^{\#} C^{+},
$$


and therefore, if $A$ is the constant of Theorem 5a,

$$
\left\|u\left(\eta_{1}, \cdot\right)-u\left(\eta_{2}, \cdot\right)\right\|_{0} \leqq A\left\|E^{+}\left(\eta_{1}+\right) E^{-}\left(\eta_{2}\right) c^{\#} C^{+}\right\|_{0} \cdot
$$

By Theorem $2 \mathrm{~d}$ there exists a partition $\pi$ of $\left(\zeta_{1}<\eta<\infty\right)$ such that if $h=c^{\#} C^{+}$ then

$$
\int_{\Delta(j)}|\boldsymbol{h}(d \xi)|<\frac{\varepsilon}{A}
$$

if $\Delta(j)$ is not a point. For such a $j$

$$
\left\|E^{+}\left(\eta_{1}+\right) E^{-}\left(\eta_{2}\right) c^{\#} C^{+}\right\|_{0} \leqq \int_{\Delta(j)}|h(d \xi)|<\frac{\varepsilon}{A} .
$$

Collecting results we see that if $\Delta(j)$ is not a point and if $\eta_{1}, \eta_{2} \in \Delta(j)$ then (1) holds. If $\Delta(j)$ is a point $\eta_{1}=\eta_{2}$ and (1) is trivial.

THEOREM 6b. Under the same assumptions there exist functions $u_{0}(\eta, \xi)$ and $v_{0}(\eta, \xi)$ defined for $\eta>\zeta_{1}, \xi \in \Xi$ such that:

(i) $u_{0}(\eta, \xi)$ and $v_{0}(\eta, \xi)$ are measurable in $\xi$ and $\eta$ for $\eta>\zeta_{1}$;

(ii) for $\eta>\zeta_{1}$ fixed, $u_{0}(\eta, \xi)$ and $v_{0}(\eta, \xi)$ are measurable in $\xi$ and $u_{0}(\eta, \xi)$ $=u_{*}(\eta, \xi), v_{0}(\eta, \xi)=v_{*}(\eta, \xi)$ except for a set of $C^{+}(d \xi)\left(C^{-}(d \xi)\right)$ measure 0.

We may suppose in addition that $\boldsymbol{u}_{0}(\eta, \xi)$ vanishes for $\xi$ not in the support of $\boldsymbol{C}^{+}(d \xi)$, that $\left|\boldsymbol{u}_{0}(\eta, \xi)\right| \leqq 1$, and that $\boldsymbol{u}_{0}(\eta, \xi)=0$ if $\xi<0$ or $\xi>\eta$. Parallel statements hold for $v_{0}(\eta, \xi)$.

Proof. For each $\varepsilon>0$ let $\pi(\varepsilon)=\{\Delta(\varepsilon, j)\}$ be a partition of $\left(\zeta_{1}, \infty\right)$ as in Theorem 6a. Choose a point $\eta(\varepsilon, j)$ in each $\Delta(\varepsilon, j)$ and set

$$
\boldsymbol{u}_{*}(\varepsilon ; \eta, \xi)=\boldsymbol{u}_{*}(\eta(\varepsilon, j), \xi) \chi(\eta, \xi), \quad \eta \in \Delta(\varepsilon, j), \xi \in \Xi, \eta \in \Delta(\varepsilon, j) .
$$

Here $\chi(\eta, \xi)$ is 1 if $0 \leqq \xi \leqq \eta$ and 0 otherwise. Clearly $\boldsymbol{u}^{*}(\varepsilon ; \eta, \xi)$ is measurable in $\xi$ and $\eta$ for $\eta>\zeta_{1}$. We define $u_{0}(\eta, \xi)$ for $\eta>\zeta_{1}$ as

$$
\lim _{m \rightarrow \infty} u_{*}\left(2^{-m} ; \eta, \xi\right)
$$

if this limit exists and as 0 if it does not. Then $\boldsymbol{u}_{0}(\eta, \xi)$ clearly satisfies (i). Fix $n>\zeta_{1}$. Since

$$
\int_{\Xi}\left|u_{*}\left(2^{-m} ; \eta, \xi\right)-u_{*}(\eta, \xi)\right| C^{+}(d \xi) \leqq 2^{-m}
$$

it follows by a familiar lemma, see A. C. Zaanen, Linear analysis, Amsterdam and Groningen, 1953 , p. 73, that (ii) holds as well. The remaining properties for $u_{0}(\eta, \xi)$ are automatic, etc.

COROllary 6c. Under the same assumptions $d(\eta)^{2}$ is measurable for $\eta>\zeta_{1}$.

Proof. Since $d(\eta)^{2}=u(\eta,\{0\})=u_{0}(\eta, 0) C^{+}(\{0\}), d(\eta)^{2}$ is measurable. 
By Corollary 5c

$$
\left|d(\eta)^{2}-d^{2}\right| \leqq A\|u(\eta, \cdot)-U(\cdot)\|_{0}
$$

and therefore

$$
\lim _{\eta \rightarrow \infty} d(\eta)^{2}=d^{2} \neq 0
$$

It now follows that there exists a $\zeta_{1}^{\prime \prime}>\zeta_{1}$ and a $D>0$ such that if $d(\eta)$ is chosen "correctly" then:

$$
d(\eta) \text { is measurable for } \eta>\zeta_{1}^{\prime \prime} \text {; }
$$

$$
\begin{gathered}
D<|d(\eta)|<D^{-1}, \quad \eta>\zeta_{1}^{\prime \prime} ; \\
\lim _{\eta \rightarrow \infty} d(\eta)=d .
\end{gathered}
$$

7. Expansions in Szegö functions. Let $c(\theta) \in W H[\mathscr{M}(\Theta)]$ where $\mathscr{M}(\Theta)$ is of type $\mathfrak{M}$. Then $c(\theta) \in W H\left[\mathscr{M}_{0}(\Theta)\right]$ as well. Let $\zeta_{1}$ be such that Theorem 5a holds in both the algebras $\mathscr{M}(\Theta)$ and $\mathscr{M}_{0}(\Theta)$ and let $\zeta_{1}^{\prime \prime}>\zeta_{1}$ be such that (2) of $\S 6$ is valid. The Szegö functions $\phi(\eta, \theta)$ and $\psi(\eta, \theta)$ are defined by

$$
\begin{aligned}
& \phi(\eta, \theta)=d(\eta)^{-1} v(\eta, \theta)(\eta, \theta), \\
& \psi(\eta, \theta)=d(\eta)^{-1} u(\eta, \theta)(-\eta, \theta),
\end{aligned}
$$

From [9] one would expect that if $\zeta_{2} \geqq \zeta_{1}^{\prime \prime}$ is sufficiently large and if $f(\theta) \in E^{+}\left(\zeta_{2}+\right) \mathscr{M}(\Theta)$ then it should be possible to represent $f(\theta)$ in the form

$$
f(\theta)=E^{+}\left(\zeta_{2}+\right) \int_{\zeta_{2}+}^{\infty} \phi(\eta, \theta) f^{\sim}(d \eta) .
$$

In the case when $\Theta$ is compact $f^{\sim}$ can be computed from $f(\theta)$ by means of a simple and elegant formula. In the present more general situation we will show that the representation (2) is possible and is unique. However, I have not, except in a quite special case, see $\S 10$, been able to give a simple formula for $\boldsymbol{f}^{\sim}$ in terms of $f$.

The assumptions made at the beginning of this section will be considered to be in force throughout.

THEOREM 7a. Let $c(\theta) \in W H[\mathscr{M}(\Theta)]$ where $\mathscr{M}(\Theta)$ is of type $\mathfrak{S}$, etc., and let $f^{\sim}(d \eta) \in E^{+}\left(\zeta_{1}^{\prime \prime}+\right) \mathscr{M}(\Xi)$. Then if $f(\theta)$ is defined by

$f(\theta)$ belongs to $E^{+}(0) \mathscr{M}(\Theta)$,

$$
f(\theta)=\int \phi(\eta, \theta) f^{\sim}(d \eta)
$$

$$
\|f\| \leqq D\left\|C^{-}\right\|\left\|f^{\sim}\right\|
$$

where $D$ is defined by (2) of $\S 6$, and if $f=\mathscr{F}^{-1} f, B \in \mathscr{B}, B \geqq 0$, then 


$$
\boldsymbol{f}(B)=\int_{\eta+\xi \in B} \int_{\boldsymbol{B}} d(\eta)^{-1} v_{0}(\eta, \xi) C^{-}(d \xi) \boldsymbol{f}^{\sim}(d \eta) .
$$

Proof. Let $\theta$ be fixed. We have

$$
\phi(\eta, \theta)=d(\eta)^{-1}(\eta, \theta) \int(\xi, \theta) v_{0}(\eta, \xi) C^{-}(d \xi)
$$

This shows that $\phi(\eta, \theta)$ is measurable in $\eta$ and further that $|\phi(\eta, \theta)| \leqq D\left\|C^{-}\right\|_{0}$. Thus $f(\theta)$ is well defined for $\theta \in \Theta$. We have

$$
f(\theta)=\int d(\eta)^{-1}(\eta, \theta) f^{\sim}(d \eta) \int(\xi, \theta) v_{0}(\eta, \xi) C^{-}(d \xi) .
$$

The integrand of this iterated integral is measurable in $\eta$ and $\xi$, the integration is extended over the product of the support of $\boldsymbol{f}^{\sim}$ with the support of $\boldsymbol{C}^{-}$which is $\sigma$-finite, and the integral is absolutely convergent. We may therefore apply Fubini's theorem to obtain

$$
f(\theta)=\iint d(\eta)^{-1} v_{0}(\eta, \xi)(\xi+\eta, \theta) C^{-}(d \xi) f^{\sim}(d \eta),
$$

from which (4) follows immediately. Since $\left|v_{0}(\eta, \xi)\right| \leqq 1$ we have

$$
|\boldsymbol{f}(B)| \leqq D \int_{\xi+\eta \in B} \int_{\boldsymbol{B}^{-}} \boldsymbol{C}^{-}(d \xi)\left|\boldsymbol{f}^{\sim}(d \eta)\right|,
$$

and thus

which implies (3).

$$
\boldsymbol{f}<<D\left[\boldsymbol{C}^{-} *\left|\boldsymbol{f}^{\sim}\right|\right]
$$

LEMMA 7b. Let $c \in W H[\mathscr{M}(\Theta)]$ where $\mathscr{M}(\Theta)$ is of type $\mathfrak{M}$, etc. For any $\zeta \geqq 0$ we define

$$
\Lambda(\zeta) f=d^{-1} E^{+}(\zeta+) V f, \quad f \in E^{+}(\zeta+) \mathscr{M}(\Theta)
$$

Then $\Lambda(\zeta)$ has an inverse given by the formula

$$
\Lambda(\zeta)^{-1} f=d E^{+}(\zeta+) V^{-1} f
$$

Proof. Let $f \in E(\zeta+) \mathscr{M}(\Theta)$. Then

$$
E^{+}(\zeta+) V^{-1} E^{+}(\zeta+) V f=E^{+}(\zeta+) V^{-1}\left[V f-E^{-}(\zeta) V f\right]=E^{+}(\zeta+) f=f,
$$

and similarly

$$
E^{+}(\zeta+) V E^{+}(\zeta+) V^{-1} f=f .
$$

LEMMA 7c. Let $c \in W H[\mathscr{M}(\Theta)]$ where $\mathscr{M}(\Theta)$ is of type $\subseteq$, etc. For $\zeta>\zeta_{1}^{\prime \prime}$ let $\Delta(\zeta) f^{\sim}=f$ be the mapping of $E^{+}(\zeta+) \mathscr{M}(\Theta)$ into itself defined by

$$
f(B)=\iint_{\xi+\eta \in B} d(\eta)^{-1} v_{0}(\eta, \xi) C^{-}(d \xi) f^{\sim}(d \eta),
$$


for $B>\zeta$. Then given $\varepsilon>0$ there exists $\zeta_{2} \geqq \zeta_{1}^{\prime \prime}$ such that if $\zeta \geqq \zeta_{2}$

$$
\|\Lambda(\zeta)-\Delta(\zeta)\| \leqq \varepsilon \text {. }
$$

Proof. It is evident that we can write $V(d \xi)$ in the form

$$
\boldsymbol{V}(d \xi)=V_{0}(\xi) C^{-}(d \xi)
$$

where $V_{0}(\xi)$ is measurable, etc. If $B>\zeta$

$$
\begin{aligned}
\{\Lambda(\zeta)-\Delta(\zeta)\} \boldsymbol{f}^{\sim}(B) & =\int_{\xi+\eta \epsilon^{\prime} B}\left\{d^{-1} V_{0}(\xi)-d(\eta)^{-1} v_{0}(\eta, \xi)\right\} C^{-}(d \xi) f^{\sim}(d \eta) \\
& =I_{1}+I_{2}
\end{aligned}
$$

where

$$
\begin{aligned}
& I_{1}=\int_{\xi+\eta \in B} d^{-1}\left[V_{0}(\xi)-v_{0}(\eta, \xi)\right] C^{\sim}(d \xi) f^{-}(d \eta), \\
& I_{2}=\int_{\xi+\eta \in B}\left[d^{\sim 1}-d(\eta)^{-1}\right] v_{0}(\eta, \xi) C^{-}(d \xi) f^{\sim}(d \eta) .
\end{aligned}
$$

In these integrals $\boldsymbol{f}^{\sim}(d \eta)$ has no mass in $-\infty \leqq \eta<\zeta$. By (2) of Corollary 5c if $\eta>\zeta$ then

where

$$
\left|\boldsymbol{V}_{0}(\xi)-\boldsymbol{v}_{0}(\eta, \xi)\right| \boldsymbol{C}^{-}(d \xi)<<\boldsymbol{R}_{\zeta}(d \xi)
$$

$$
R_{\zeta}=2 C^{-} * E^{-}(-\zeta-)\left[C^{-} * c^{\#}\right] .
$$

Here $c^{\#}$ stands for the measure $|c(d \xi)|$. Let

We have

$$
r_{\zeta}=\underset{\eta>\zeta}{\text { l.u.b. }}\left|d^{-1}-d(\eta)^{-1}\right|
$$

and since

$$
[\Lambda(\zeta)-\Delta(\zeta)] f^{\sim}<<\left[R_{\zeta}+r_{\zeta} C^{-}\right] *\left[f^{\sim}\right]^{*}
$$

$$
\lim _{\zeta \rightarrow \infty}\left\|R_{\zeta}\right\|=\lim _{\zeta \rightarrow 0} r_{\zeta}=0,
$$

our desired result follows.

THEOREM 7d. Let $\mathscr{M}(\Theta)$ be of type $\subseteq$ and let $c \in W H[\mathscr{M}(\Theta)]$, etc. Then there exists $\zeta_{2} \geqq \zeta_{1}^{\prime \prime}$ such that if $f(\theta) \in E^{+}\left(\zeta_{2}+\right) \mathscr{M}(\Theta)$ then

$$
f(\theta)=E^{+}\left(\zeta_{2}+\right) \int \phi(\eta, \theta) f^{\sim}(d \eta),
$$

where $\boldsymbol{f}^{\sim} \in \boldsymbol{E}^{+}\left(\zeta_{2}+\right) \mathscr{M}(\Xi)$ and where

$$
A_{1}\|f\| \leqq\|f \sim\| \leqq A_{2}\|f\|
$$


Here $A_{1}$ and $A_{2}$ are finite positive constants independent of $f$. Similarly if $g(\theta) \in E^{-}\left(-\zeta_{2}-\right) \mathscr{M}(\Theta)$ then

$$
g(\theta)=E^{-}\left(-\zeta_{2}-\right) \int \psi(\eta, \theta) g^{\sim}(d \eta)
$$

where $\mathrm{g}^{\sim} \in \boldsymbol{E}^{-}\left(-\zeta_{2}-\right) \mathscr{M}(\Xi)$ and where

$$
A_{1}\|g\| \leqq\|g \sim\| \leqq A_{2}\|g\| \text {. }
$$

Proof. This follows from Lemmas $7 \mathrm{~b}$ and $7 \mathrm{c}$ together with the result that in any Banach algebra (not necessarily commutative) any element "near enough" to an invertible element is itself invertible. See the reference in $\$ 5$.

THEOREM 7e. Let $\mathscr{M}(\Theta)$ be of type $\mathfrak{M}$ and let $c \in W H[\mathscr{M}(\Theta)]$, etc. Then there exists $\zeta_{2}>\zeta_{1}^{\prime \prime}$ such that if $f(\theta) \in E^{+}\left(\zeta_{2}+\right) \mathscr{M}(\Theta)$

$$
f(\theta)=E^{+}\left(\zeta_{2}+\right) \int \phi(\eta, \theta) f^{\sim}(d \eta)
$$

where $f^{\sim} \in E^{+}\left(\zeta_{2}+\right) \mathscr{M}_{0}(\Xi)$, and $f^{\sim}$ is unique. Similarly if $g(\theta) \in E^{\sim}\left(-\zeta_{2}-\right) \mathscr{M}(\Theta)$ then

$$
g(\theta)=E^{-}\left(-\zeta_{2}-\right) \int \psi(\eta, \theta) g^{\sim}(d \eta)
$$

where $\boldsymbol{g}^{\sim} \in \boldsymbol{E}^{-}\left(-\zeta_{2}-\right) \mathscr{M}_{0}(\Xi)$ and $\boldsymbol{g}^{\sim}$ is unique.

Proof. This follows from the fact that $\mathscr{M}(\Theta) \subset \mathscr{M}_{0}(\Theta)$. Note that if $\mathscr{M}(\Theta)$ is only of type $\mathfrak{M}$ we cannot assert that $\boldsymbol{f}^{\sim} \in \mathscr{M}(\Xi)$, but only that $\boldsymbol{f}^{\sim} \in \mathscr{M}_{0}(\Xi)$.

We will need the following lemma.

LeMMA 7f. Let $g(\theta) \in \mathscr{M}_{0}(\Theta), h(d \xi) \in \mathscr{M}_{0}(\Xi)$; then

$$
E^{+}(\zeta)\left[g(\theta) \int(\xi, \theta) h(d \xi)\right]=\int\left[E^{+}(\zeta) g(\theta)(\xi, \theta)\right] h(d \xi) .
$$

Let $c \in W H\left[\mathscr{M}_{0}(\Xi)\right]$, etc., and let $\tilde{f}^{\sim}(d \xi) \in E^{+}\left(\zeta_{2}+\right) \mathscr{M}_{0}(\Xi) ;$ then if $\zeta>\zeta_{2}$

$$
E^{+}(\zeta)\left[g(\theta) \int \phi(\xi, \theta) f^{\sim}(d \xi)\right]=\int\left[E^{+}(\zeta) g(\theta) \phi(\xi, \theta)\right] f^{\sim}(d \xi),
$$

Proof. Note $E^{+}(\zeta)$ can be replaced by $E^{+}(\zeta+), E^{-}(\zeta)$ or $E^{-}(\zeta-)$ in (10) and (11). Also there is a parallel result in which $\phi$ is replaced by $\psi$. We will only prove (11) since (10) is similar but simpler. By Theorem 7a (applied to $\mathscr{M}_{0}(\Theta)$ )

$$
\begin{aligned}
\int \phi(\eta, \theta) \boldsymbol{f}^{\sim}(d \eta) & =\iint d(\eta)^{-1}(\xi+\eta, \theta) \boldsymbol{v}_{0}(\eta, \xi) \boldsymbol{C}^{-}(d \xi) \boldsymbol{f}^{\sim}(d \eta), \\
g(\theta) \int \phi(\eta, \theta) \boldsymbol{f}^{\sim}(d \eta) & =\int(\omega, \theta) g(d \omega) \iint d(\eta)^{-1}(\xi+\eta, \theta) \boldsymbol{v}_{0}(\eta, \xi) \boldsymbol{C}^{-}(d \xi) \boldsymbol{f}^{\sim}(d \eta), \\
& =\iiint d(\eta)^{-1}(\omega+\xi+\eta, \theta) v_{0}(\eta, \xi) g(d \omega) \boldsymbol{C}^{-}(d \xi) \boldsymbol{f}^{\sim}(d \eta),
\end{aligned}
$$


and consequently

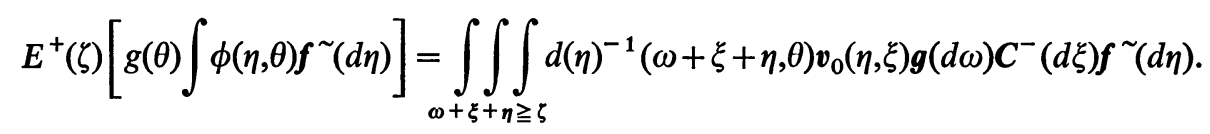

On the other hand,

$$
\begin{aligned}
g(\theta) \phi(\eta, \theta) & =\iint d(\eta)^{-1}(\omega+\xi+\eta, \theta) v_{0}(\eta, \xi) g(d \omega) C^{-}(d \xi), \\
E^{+}(\zeta)[g(\theta) \phi(\eta, \theta)] & =\iint_{\omega+\xi+\eta \geq \zeta} d(\eta)^{-1}(\omega+\xi+\eta, \theta) v_{0}(\eta, \xi) g(d \omega) C^{-}(d \xi), \\
\int E^{+}(\zeta)[g(\theta) \phi(\eta, \theta)] f^{\sim}(d \eta) & =\iiint_{\omega+\xi+\eta \geqq \zeta} d(\eta)^{-1}(\omega+\xi+\eta, \theta) v_{0}(\eta, \xi) g(d \omega) C^{-}(d \xi) \boldsymbol{f}^{\sim}(d \eta) .
\end{aligned}
$$

THEOREM 7g. Let $\mathscr{M}(\Theta)$ be of type $\mathfrak{M}$ and let $c(\theta) \in W H[\mathscr{M}(\Theta)]$, etc. For $f(\theta) \in E^{+}(0) \mathscr{M}(\Theta)$ we define

$$
f(\eta, \theta)=f(\theta)-\int_{\eta^{+}}^{\infty} \phi(\xi, \theta) f^{\sim}(d \xi), \quad \eta>\zeta_{2} .
$$

Then $f(\eta, \theta) \in E^{+}(0) E^{-}(\eta) \mathscr{M}(\Theta)$ and

$$
W_{c}^{+}(\eta) f(\eta)=W_{c}^{+}(\eta) f .
$$

Proof. This result was communicated to me by Professor Baxter. It follows from Theorem 7e that $f(\eta, \theta) \in E^{+}(0) E^{-}(\eta) \mathscr{M}(\Theta)$. To derive (13) multiply (12) through by $c(\theta)$, apply $E^{+}(0) E^{-}(\eta)$, and use Lemma 7 f.

We can now deduce a stronger version of Theorem $7 \mathrm{e}$.

THEOREM $7 \mathrm{~h}$. Let $\mathscr{M}(\Theta)$ be of type $\mathfrak{M}$ and let $c \in W H[\mathscr{M}(\Theta)]$, etc. If $f \in E^{+}\left(\zeta_{2}+\right) \mathscr{M}(\Theta)$ then

$$
\lim _{\eta \rightarrow+\infty}\left\|f(\theta)-E^{+}\left(\zeta_{2}+\right) \int_{\zeta_{2}+}^{\eta} \phi(\xi, \theta) f^{\sim}\right\|(d \xi)=0 .
$$

Proof. We have

By (13) and Corollary 5c

$$
\begin{aligned}
f(\theta)-E^{+}\left(\zeta_{2}+\right) \int_{\zeta_{2}+}^{\eta} \phi(\xi, \theta) \boldsymbol{f}^{\sim}(d \xi) & =\int_{\eta^{+}}^{\infty} \phi(\xi, \theta) \boldsymbol{f}^{\sim}(d \xi) \\
& =f(\theta)-f(\eta, \theta) .
\end{aligned}
$$

$$
\|f(\eta, \theta)-f(\theta)\| \leqq A\left\|E^{+}(\eta+) f\right\|
$$

where $A$ depends only upon $c$.

It is of considerable interest that a very simple and explicit closed formula can be given for $f(\eta, \theta)$ defined by (12). This result is closely related to the 
Christoffel-Darboux formula for orthogonal polynomials. The reader interested in this relationship should consult $[7, \S 5]$. For $\eta>\zeta_{1}^{\prime \prime}$ we set

$$
\begin{aligned}
S^{+}(\eta) f \cdot(\theta)=d(\eta)^{-2}\left[u(\eta, \theta) E^{+}(0)\right. & \{f(\theta) c(\theta) v(\eta, \theta)\} \\
& \left.-v(\eta, \theta) E^{+}(\eta+)\{f(\theta) c(\theta) u(\eta, \theta)\}\right] .
\end{aligned}
$$

THEOREM 7i. Let $\mathscr{M}(\Theta)$ be of type $\mathfrak{M}$ and let $c \in W H[\mathscr{M}(\Theta)]$, etc. For $f(\theta) \in E^{+}(0) \mathscr{M}(\Theta)$ let $f(\eta, \theta)$ be defined by $(12)$; then

$$
f(\eta, \theta)=S^{+}(\eta) f \cdot(\theta)
$$

Proof. It can be verified that if $\eta>\xi_{2}$ then

$$
S^{+}(\eta)(\xi, \theta)=(\xi, \theta), \quad 0 \leqq \xi \leqq \eta,
$$

and

$$
S^{+}(\eta) \phi(\xi, \theta)=0, \quad \eta<\xi ;
$$

see [9]. Apply $S^{+}(\eta)$ to both sides of (12), use Lemma 7f, and the relations $\left(15^{\prime}\right)$ and $\left(15^{\prime \prime}\right)$.

This identity is closely related to Theorem 5a.

8. Recursion formulas. By Theorem 7e if $c(\theta) \in W H[\mathscr{M}(\Theta)]$ where $\mathscr{M}(\Theta)$ is of type $\mathfrak{M}$, then

(1)

$$
U(\theta)=\int_{\zeta_{2}+}^{\infty} \phi(\xi, \theta) U^{\sim}(d \xi)+U^{\prime \prime}(\theta)
$$

$$
V(\theta)=\int_{\zeta_{2}+}^{\infty} \psi(\xi, \theta) V^{\sim}(d \xi)+V^{\prime \prime}(\theta),
$$

where $U^{\prime \prime}(\theta) \in E^{+}(0) E^{-}\left(\zeta_{2}\right) \mathscr{M}(\Theta), V^{\prime \prime}(\theta) \in E^{-}(0) E^{+}\left(-\zeta_{2}\right) \mathscr{M}(\Theta)$. We define

$$
\begin{aligned}
& \boldsymbol{\alpha}(d \xi)=d(\xi)^{-1} \boldsymbol{U}^{\sim}(d \xi), \\
& \boldsymbol{\beta}(d \xi)=d(\xi)^{-1} \boldsymbol{V}^{\sim}(d \xi),
\end{aligned}
$$

In terms of the $\alpha$ 's and the $\beta$ 's we have the following basic recursion formulas.

THEOREM 8a. Let $\mathscr{M}(\Theta)$ be of type $\mathfrak{M}$, and let $c(\theta) \in W H[\mathscr{M}(\Theta)]$, etc. If $\eta_{2}>\eta_{1}>\zeta_{2}$ then

$$
u\left(\eta_{2}, \theta\right)-u\left(\eta_{1}, \theta\right)=\int_{\eta_{1}<\xi \leqq \eta_{2}} v(\xi, \theta)(\xi, \theta) \alpha(d \xi),
$$

$$
v\left(\eta_{2}, \theta\right)-v\left(\eta_{1}, \theta\right)=\int_{\eta_{1}<\xi \leqq \eta_{2}} u(\xi, \theta)(-\xi, \theta) \boldsymbol{\beta}(d \xi)
$$


Proof. This is an immediate consequence of (1) and Theorem $7 \mathrm{~g}$.

THEOREM 8b. Under the same assumptions.

$$
\begin{aligned}
d\left(\eta_{2}\right)^{2}-d\left(\eta_{1}\right)^{2} & =\int_{\eta_{1}<\xi \leqq \eta_{2}} d(\xi)^{2} \boldsymbol{\beta}(\{\xi\}) \alpha(d \xi) \\
& =\int_{\eta_{1}<\xi \leqq \eta_{2}} d(\xi)^{2} \alpha(\{\xi\}) \beta(d \xi) .
\end{aligned}
$$

Proof. Apply $E^{-}\left(-\eta_{2}\right)$ to both sides of the second equation of (2). Using Lemma $7 \mathrm{f}$ we see that (writing $\xi$ for $\eta_{2}$ )

$$
\boldsymbol{v}(\xi,-\{\xi\})=\boldsymbol{u}(\xi,\{0\}) \boldsymbol{\beta}(\{\xi\})
$$

Apply $E^{-}(0)$ to both sides of the first equation of (2). If $\eta_{2}>\eta_{1}>\zeta_{2}$ we obtain

$$
u\left(\eta_{2},\{0\}\right)-u\left(\eta_{1},\{0\}\right)=\int_{\eta_{1}<\xi \leqq \eta_{2}} v(\xi,-\{\xi\}) \alpha(d \xi) .
$$

Inserting the first formula in the second gives the first formula of (3), etc.

COROLLARY 8c. Under the same assumptions

$$
\boldsymbol{\beta}(\{\xi\}) \alpha(\{\xi\}) \neq 1, \quad \xi>\zeta_{2} .
$$

Proof. Suppose that for some $\eta_{2}>\zeta_{2}, \boldsymbol{\beta}\left(\left\{\eta_{2}\right\}\right) \alpha\left(\left\{\eta_{2}\right\}\right)=1$. It follows from (3) that if $\zeta_{2}<\eta_{1}<\eta_{2}$

$$
-d\left(\eta_{1}\right)^{2}=\int_{\eta_{1}<\xi<\eta_{2}} d(\xi)^{2} \beta(\{\xi\}) \alpha(d \xi)
$$

which implies that

$$
\lim _{\eta_{1} \rightarrow \eta_{2}^{-}} d\left(\eta_{1}\right)^{2}=0 .
$$

Since $d\left(\eta_{1}\right)^{2} \geqq D^{-1}>0$ for $\eta_{1}>\zeta_{2} \geqq \zeta_{1}^{\prime \prime}$ this is a contradiction.

9. Product integration. Using the theory of product integration we can draw some interesting conclusions from Theorems $8 \mathrm{a}$ and $8 \mathrm{~b}$. We begin by stating a few definitions and some standard theorems. For demonstrations of similar results see [5].

Let

$$
A=[a(i, j)], \quad i, j=1, \cdots, N,
$$

be an $N \times N$ matrix with complex entries. We define the norm of $A,|A|$, by

$$
|A|=N \max _{i, j=1, \ldots, N}|a(i, j)| .
$$

It is easily seen that

$$
|a A+b B| \leqq|a||A|+|b||B|,
$$


and

$$
|A B| \leqq|A||B|
$$

Let

$$
A(d \xi)=[a(i, j ; d \xi)], \quad i, j=1, \cdots, N,
$$

be an $N \times N$ matrix whose entries are elements in $M_{0}(\Xi)$. Thuis will be indicated by writing $A(d \xi) \in M_{0}(\Xi)$. We define

$$
\int A(d \xi)=\left[\int a(i, j ; d \xi)\right]
$$

If $B$ is any Borel set we define

$$
|A|_{B}=\int_{B}|[a(i, j ; d \xi)]|
$$

Note that $|A(d \xi)|=N \max _{i, j=1, \ldots, N}|a(i, j ; d \xi)|$ is well defined, and that

$$
\left|\int_{B} A(d \xi)\right| \leqq|A|_{B}
$$

Corresponding to any partition $\pi=\{\Delta(j)\}$, of $\Xi$ we define

$$
S_{\pi}^{n}(A)=\prod_{j}\left[I+\int_{\Delta(j)} A(d \xi)\right]
$$

Given two partitions $\pi=\{\Delta(i)\}_{i}$ and $\pi^{\prime}=\left\{\Delta^{\prime}(j)\right\}_{j}$ we say that $\pi^{\prime}$ is a refinement of $\pi, \pi^{\prime}<\pi$, if every $\Delta^{\prime}(j)$ is included in some one $\Delta(i)$. Under the relation " $<$ ", the set of all partitions of $\Xi$ becomes a directed set. If in the topology defined by the norm (1) the net $S_{n}^{n}(A)$ has a limit, denoted by

$$
\hat{\int} A(d \xi),
$$

this limit is called the "product integral" of $\boldsymbol{A}$.

THEOREM 9a. If $A(d \xi) \in \mathscr{M}_{0}(\Xi)$ then the product integral

$$
\int A(d \xi)
$$

exists.

$$
\begin{aligned}
& \text { If }-\infty \leqq \xi_{1} \leqq \xi_{2} \leqq \infty \text { we define } \\
& \int_{\xi_{1}}^{\xi_{2}} A(d \xi)=\hat{\int} A\left(\xi_{1}, \xi_{2} ; d \xi\right)
\end{aligned}
$$

where $A\left(\xi_{1}, \xi_{2} ; d \xi\right)$ is the restriction of $A(d \xi)$ to $\left[\xi_{1}, \xi_{2}\right]$. It is clear that instead of $\left[\xi_{1}, \xi_{2}\right]$ we could use $\left(\xi_{1}, \xi_{2}\right]$, etc. It is also evident that if $\xi_{1}<\xi_{2}<\xi_{3}$ 


$$
\left\{\int_{\xi_{1}+}^{\xi_{2}} A(d \xi)\right\} \quad\left\{\int_{\xi_{2}+}^{\xi_{3}} A(d \xi)\right\}=\int_{\xi_{1}+}^{\xi_{3}} A(d \xi) .
$$

Lemma 9b. Let $A(d \xi) \in \mathscr{M}_{0}(\Xi)$ and let

$$
\boldsymbol{B}(\zeta)=\int_{\zeta+}^{\infty} A(d \xi) .
$$

Then $\boldsymbol{B}(\zeta)$ is a measurable matrix function and

$$
|\boldsymbol{B}(\zeta)| \leqq \exp [|A| \Xi], \quad \zeta \in \Xi \text {. }
$$

Let $X$ be an $N \times 1$ matrix-this is a column vector. For $\zeta$ fixed we set

$$
X(\eta)=\left\{\int_{\eta^{+}}^{\zeta} A(d \xi)\right\} X, \quad \eta<\zeta,
$$

and

$$
\boldsymbol{X}(\zeta)=X
$$

THEOREM 9c. Under the above assumptions

$$
\boldsymbol{X}(\eta)-\boldsymbol{X}(\zeta)=\int_{\eta+}^{\zeta} A(d \xi) X(\xi), \quad n<\zeta .
$$

We also have the following converse result.

THEOREM 9d. Let $X(\xi)$ be an $N \times 1$ complex valued matrix defined for $\zeta_{1} \leqq \xi<\infty$. We assume that $\mathrm{X}(\xi)$ is uniformly bounded and measurable. Let $A(d \xi) \in \mathscr{M}_{0}(\Xi)$ and let

$$
X(\eta)-X\left(\zeta_{2}\right)=\int_{\eta^{+}}^{\zeta_{2}} A(d \xi) X(\xi)
$$

for $\zeta_{1} \leqq \eta<\infty$. Then

$$
X(\eta)=\left[\int_{\eta^{+}}^{\zeta_{2}} A(d \xi)\right] \boldsymbol{X}\left(\xi_{2}\right), \quad \zeta_{1} \leqq \eta<\xi_{2}<\infty .
$$

Theorems $8 \mathrm{a}$ and $8 \mathrm{~b}$ combined with Theorem 9d yield the following results.

THEOREM 9e. Let $\mathscr{M}(\Theta)$ be of type $\mathfrak{M}$ and let $c \in W H[\mathscr{M}(\Theta)]$. If $\zeta_{2}<\eta_{1}<\eta_{2}$ then

$$
\left[\begin{array}{l}
u\left(\eta_{1}, \theta\right) \\
v\left(\eta_{1}, \theta\right)
\end{array}\right]=\left\{\int_{\eta_{1}+}^{\eta_{2}}\left[\begin{array}{cc}
0 & -\alpha(d \xi)(\xi, \theta) \\
-\beta(d \xi)(-\xi, \theta) & 0
\end{array}\right]\right\}\left[\begin{array}{l}
u\left(\eta_{2}, \theta\right) \\
v\left(\eta_{2}, \theta\right)
\end{array}\right] .
$$

THEOREM 9f. Under the same assumptions

$$
d\left(\eta_{1}\right)^{2}=\left\{\prod_{\eta_{1}<\xi \leqq \eta_{2}}[1-\alpha(\{\xi\}) \beta(\{\xi\})]\right\} d\left(\eta_{2}\right)^{2} .
$$


Here the product is extended over the at most countable set of values $\xi$ for which $\alpha(\{\xi\}) \boldsymbol{\beta}(\{\xi\})$ is not zero.

10. The inversion formula. Suppose that $\Theta$ is compact. We will assume $\Theta$ has Haar measure 1 . Let $c \in W H[\mathscr{M}(\Theta)]$ where $\mathscr{M}(\Theta)$ is of type $\mathfrak{M}$. It is easily verified that if $\eta_{1}, \eta_{2}>\zeta_{1}^{\prime}$ then

$$
\int_{\Theta} \phi\left(\eta_{1}, \theta\right) \psi\left(\eta_{2}, \theta\right) c(\theta) d \theta=\delta\left(\eta_{1}, \eta_{2}\right)
$$

where $\delta\left(\eta_{1}, \eta_{2}\right)$ is 1 if $\eta_{1}=\eta_{2}$ and is 0 otherwise. We also note that if $\eta>\zeta_{1}^{\prime}$ and $g(\theta) \in[0, \eta)$ then

$$
\int_{\theta} g(\theta) \psi(\eta, \theta) c(\theta) d \theta=0 .
$$

See $[9, \S 6]$. By Theorem 7e if $f(\theta) \in E^{+}(0) \mathscr{M}(\Theta)$ then

$$
f(\theta)=f_{1}(\theta)+\int_{\zeta_{2}+}^{\infty} \phi(\eta, \theta) f^{\sim}(d \eta)
$$

where $f_{1}(\theta) \in E^{+}(0) E^{-}\left(\zeta_{2}\right) \mathscr{M}(\Theta)$. Since $\Xi$ is discrete, functions, and measures are the same and this formula can, using an evident notation, be rewritten in the form

$$
f(\theta)=f_{1}(\theta)+\sum_{\eta>\zeta_{2}} \phi(\eta, \theta) \boldsymbol{f}^{\sim}(\eta) .
$$

Multiplying (3) by $\psi(\xi, \theta) c(\theta)$ where $\xi>\zeta_{2}$, integrating over $\Theta$, and using (1) and (2) we find that

$$
\boldsymbol{f}^{\sim}(\xi)=\int_{\Theta} f(\theta) \psi(\xi, \theta) c(\theta) d \theta
$$

(There is of course a parallel result for expansions using $\psi$ 's.) The formula (4) plays a basic role in the exposition of the case $\Theta$ compact which is given in [9].

In the development which we have given of the general case, $\Theta$ locally compact, we have been able to proceed without using the analogue of this formula. Curiously enough it is not at all clear what the correct analogue of (4) is for $\Theta$ not compact. However, in one important case we can give a formula for $f^{\sim}$ in terms of $f$ or, what is the same thing, in terms of $\boldsymbol{f}$, which can be considered as an analogue of (4). We assume henceforth that $\Theta$ is not compact. Let $\mathscr{A}_{0}(\Xi)$ be the subalgebra of $\mathscr{M}_{0}(\Xi)$ consisting of those measures $c(d \xi)$ of the form

$$
c(d \xi)=c_{0}(\xi) d \xi+c(\{0\}) \delta(d \xi)
$$

where $c_{0}(\xi)$ is in $L^{1}(\Xi)$ and where $\delta(d \xi)$ is the Dirac measure with unit mass at 
$\xi=0$. By convention $A(\Xi)$ will always denote a subalgebra of $A_{0}(\Xi)$, etc. By the Riemann-Lebesgue theorem if $c \in \mathscr{A}_{0}(\Xi)$ then

$$
\lim _{\theta \rightarrow \infty} c(\theta)=c(\{0\})=d^{-2} ;
$$

see (4), §4. Suppose that $A(\Theta)$ is of type $\mathfrak{M}$, and that $c \in W H[A(A(\Theta)]$, etc. If $\eta>\zeta_{1}^{\prime}$, then since

$$
\begin{aligned}
& c(\theta) u(\eta, \theta)=(-\infty, 0)+1+(\eta, \infty), \\
& c(\theta) v(\eta, \theta)=(-\infty,-\eta)+1+(0, \infty),
\end{aligned}
$$

it follows that

$$
c(\infty) u(\eta, \infty)=c(\infty) v(\eta, \infty) v(\eta, \infty)=1
$$

and thus that

$$
u(\eta,\{0\})=u(\eta, \infty)=v(\eta,\{\infty\})=v(\eta, \infty)=d^{2}
$$

This implies in particular that $d(\eta)^{2} \equiv d^{2}$ and we may therefore take

$$
d(\eta)=d, \quad \eta \geqq \zeta_{1} .
$$

We may also assume that $\zeta_{1}^{\prime \prime}=\zeta_{1}$. In view of Theorem $6 \mathrm{~b}$ we may, with a slight change of notation, write

$$
\begin{aligned}
& u(\eta, d \xi)=d^{2} \delta(d \xi)+u_{0}(\eta, \xi) d \xi, \\
& v(\eta, d \xi)=d^{2} \delta(d \xi)+v_{0}(\eta, \xi) d \xi
\end{aligned}
$$

where $u_{0}(\eta, \xi)$ is measurable in $\eta$ and $\xi,\left|u_{0}(\eta, \xi)\right| \leqq C^{+}(\xi)$, and $u_{0}(\eta, \xi)=0$ for $\xi \leqq 0$ and $\xi>\eta$. Parallel statements holds for $v_{0}(\eta, \xi)$.

In this case a somewhat lengthy calculation, which we omit, yields the following result.

THEOREM 10a. Let $A(\Theta)$ be of type $\mathfrak{M}$, and let $c \in W H[\mathscr{A}(\Theta)]$, etc. If $f \in E^{+}\left(\zeta_{1}+\right) A(\Theta)$ then

$$
f^{\sim}(\eta)=d\{f * c\} \cdot(\eta)+d^{-1} \int u_{0}(\eta, \eta-\zeta)\{f * c\} \cdot(\zeta) d \zeta
$$

for $\eta>\zeta_{2}$.

Note that since $\boldsymbol{f}^{\sim} \in \boldsymbol{E}^{+}\left(\zeta_{2}+\right) \mathscr{A}(\Xi), \boldsymbol{f}^{\sim}$ is absolutely continuous and we may (and do) replace $\boldsymbol{f}^{\sim}(d \xi)$ by $\boldsymbol{f}^{\sim}(\xi) d \xi$. Similarly $\boldsymbol{f}$, but not $\boldsymbol{c}$, is absolutely continuous.

There is an evident dual result in which the roles of the $\phi$ 's and $\psi$ 's are interchanged. In accordance with our usual convention we will regard Theorem 10a as including this result as well, 


\section{REFERENCES}

1. G. Baxter, Polynomials defined by a difference system, J. Math. Anal. Appl. 2 (1961), 223-263.

2. - A convergence equivalence related to polynomials on the unit circle, Trans. AmerMath. Soc. 99 (1961), 471-487.

3. —_ A norm inequality for a 'finite section' Wiener-Hopf equation, Illinois J. Math. 7 (1963), 97-103.

4. G. Baxter and I. I. Hirschman, Jr., An explicit inversion formula for finite section WienerHopf operators, Bull. Amer. Math. Soc. 70 (1964), 820-823.

5. Garrett Birkhoff, On product integration, J. Mathematical Phys. 16 (1937), 104-132.

6. U. Grenander and G. Szegö, Toeplitz forms and their applications, California Monographs in Mathematical Sciences, University of California Press, Berkeley, Calif., 1958.

7. I. I. Hirschman, Jr., Finite sections of Wiener-Hopf equations and Szegö polynomials, J. Math. Anal. Appl. 11 (1965), 290-320.

8. - - On the theorem of Szegö, Kac, and Baxter, I. Analyse Math. 14 (1965), 225-234.

9. —- Szegö polynomials on a compact group with ordered dual, Canad. J. Math. (to appear).

10. W. Rudin, Fourier analysis on groups, Interscience, New York, 1962.

11. M. Shinbrot, A class of difference kernels, Proc. Amer. Math. Soc. 13 (1962), 399-406.

WASHINGTON UNIVERSITY,

St. Louis, Missouri 